INTERVENTIONAL CARDIOLOGY AND SURGERY

\title{
Heart and heart-lung transplantation for idiopathic restrictive cardiomyopathy in children
}

\author{
M J Fenton, H Chubb, A M McMahon, P Rees, M J Elliott, M Burch
}

Heart 2006;92:85-89. doi: 10.1136/hrt.2004.049502

See end of article for authors' affiliations

......................

Correspondence to: Dr Michael Burch, Cardiothoracic Transplant, Great Ormond Street Hospital for Children, Great Ormond Street London WCIN 3JH, UK; burchm@gosh.nhs.uk

Accepted

15 November 2004

\begin{abstract}
Objective: To review the outcome of cardiac transplantation for restrictive cardiomyopathy (RCM) in children and to assess the ability of new strategies to modulate the effects of high pulmonary vascular resistance.

Design: Retrospective case note analysis of all patients receiving a transplant for RCM.

Patients: 18 children with RCM referred for transplantation assessment to Great Ormond Street Hospital, London.

Results: Eight boys and 10 girls were referred for assessment. Median age at presentation was 5.0 (mean (SD) 6.1 (4.0)l years. Fourteen orthotopic and two heterotopic transplantations were performed and two patients were referred for heart-lung transplantation. Mean duration from diagnosis to transplantation was 3.3 (3.0) years. Three patients with haemodynamic decompensation before transplantation had increased morbidity in the postoperative period. No patients died while awaiting a transplant. Three patients died in the first year after transplantation, one within 30 days. Five patients received pretransplantation prostacyclin for a mean duration of 57 (18) days. Transpulmonary gradient was reduced in four of the patients. Mean transpulmonary gradient was $27(9.8) \mathrm{mm} \mathrm{Hg}$ before and 17 (6.7) mm Hg after treatment with prostacyclin $(\mathrm{p}<0.05)$.

Conclusion: Most children with RCM require transplantation within four years of diagnosis. Referral for transplantation assessment should precede haemodynamic decompensation. Increase of pulmonary vascular resistance is a variable problem but can be modulated with pre-transplantation prostacyclin. With these strategies, orthotopic transplantation is possible in the majority of cases.
\end{abstract}

$\mathrm{R}$ estrictive cardiomyopathy (RCM) is a rare form of cardiomyopathy in childhood, accounting for between $2.5-5 \%$ of all idiopathic cardiomyopathy in that age group. ${ }^{1-3}$ It is characterised by impaired diastolic function with relatively preserved systolic function. Restriction of filling results in cardiac failure and raised pulmonary vascular resistance. ${ }^{4}$ A few series have been published documenting the clinical course after the diagnosis of RCM in childhood ${ }^{5-9}$; all have reported an accelerated deterioration in comparison with that seen in adults. Medical treatment of these patients has not shown any significant long term benefit and transplantation appears the only realistic treatment option. However, the natural history of the disease is not homogeneous and within the published series, rising pulmonary vascular resistance and clinical deterioration progress at different rates. No consistent risk factors predictive for progression have been identified.

We feel that reporting our data, the largest series of children with idiopathic RCM who have received a transplant, is helpful for such a rare condition. Furthermore, many developments in pharmacological treatment and intensive care management for pulmonary hypertension have not been reported in paediatric RCM.

\section{PATIENTS AND METHODS}

All 18 children referred to Great Ormond Street Hospital for Children over the period 1988 to 2004 are reported on. RCM was diagnosed if the children had enlarged atria and absent dilatation of the ventricles. In addition features of hypertrophic cardiomyopathy were absent and left ventricular systolic function was preserved with fractional shortening $>25 \%$ at the time of diagnosis. Patients had to exhibit neither signs of significant pericardial disease nor any primary cause of cardiomyopathy, such as metabolic disease.
The clinical course of each patient was chronicled by retrospective case note review. Variables included in the analysis were sex, age at diagnosis, time from diagnosis to transplantation, family history, shortening fraction, transpulmonary gradient (TPG) and pulmonary vascular resistance index (PVRI) at diagnosis and at the final study before transplantation, mode of transplantation, and outcome. Acute vasodilation was tested in patients at catheterisation by increasing inspired oxygen concentration and adding up to 20 parts per million of nitric oxide. Accurate assessment of PVRI and cardiac index has been possible only more recently since a mass spectrometer has been used in our department. TPG has a significant role in discussions on transplantation strategy because of its ability to reflect changes in the pulmonary vascular bed, independent of pulmonary blood flow. ${ }^{10}$ Outcome is divided into 30 day mortality and death in the first year.

Patients were divided into two groups based on complications in the perioperative period. These complications were: (1) acute graft failure requiring repeat transplantation; (2) the use of extracorporeal membrane oxygenation (ECMO) or a right ventricular assist device (RVAD); and (3) death in the first year after transplantation. The groups were compared for the period from diagnosis to transplantation and for pulmonary haemodynamic function at transplantation. Data are expressed as mean (SD). The study was granted ethical approval by the local ethical review board.

Abbreviations: ASD, atrial septal defect; ECMO, extracorporeal membrane oxygenation; PVRI, pulmonary vascular resistance index; $R M C$, restrictive cardiomyopathy; RVAD, right ventricular assist device; TPG, transpulmonary gradient; WU, Wood units 


\section{RESULTS}

The median age at diagnosis was 5.0 (mean 6.1 (4.0) years (range 1.0-14.3 years). Patients presented with a variety of common paediatric symptoms, including chest infections, heart failure, poor growth, chest pain, and vomiting. One patient presented through familial screening and four patients had a family history of cardiomyopathy. At the time of diagnosis, all patients had normal systolic function on echocardiography except one, who had a near normal shortening fraction. All patients had biatrial enlargement.

All patients received medication appropriate for management of heart failure before referral for transplantation. Five patients were also taking warfarin with no thromboembolic events after warfarin treatment. One patient had a left hemiparesis in the early weeks after diagnosis. Another patient developed complete heart block after diagnosis and presented with syncope. A pacemaker was inserted resulting in no further syncopal episodes.

\section{Progression of pulmonary hypertension}

Cardiac catheterisation was performed in all patients (table 1). The mean TPG at diagnosis and at the last study before transplantation were $18.5 \quad$ (11.3) and 13.2 (9.3) $\mathrm{mm} \mathrm{Hg}$, respectively.

Alternative strategies to orthotopic transplantation were considered when the TPG was greater than $14 \mathrm{~mm} \mathrm{Hg}$ and PVRI was greater than 6 Wood units $(\mathrm{WU}) \cdot \mathrm{m}^{2}$. One patient received an orthotopic transplant while being bridged to transplantation with ECMO outside these parameters due to being unlikely to survive the extended waiting time for heart-lung transplantation. Two transplantations were heterotopic and two patients are awaiting heart-lung transplantation. The development of this severe pulmonary hypertension was not related to any investigated pretransplantation characteristics. The TPG/PVRI increased greatly in only two patients during the follow up period. We noticed TPG fall in one patient from $23 \mathrm{~mm} \mathrm{Hg}$ to $6 \mathrm{~mm} \mathrm{Hg}$. An atrial septal defect (ASD) was closed between these two measurements.

\section{Duration from diagnosis to transplantation}

The mean time from diagnosis to transplantation was 40 (35.5) months (range 1-107 months). This was not related either to the TPG or to complications in the post-transplantation period.

\section{Haemodynamic compromise}

Left ventricular function deteriorated in six patients by the time of transplantation, accompanied by worsening symptoms of cardiac failure. Three patients developed severe haemodynamic compromise: one patient required inotropic support and two required bridging to orthotopic cardiac transplantation with ECMO.

\section{Pre-treatment with prostacyclin}

Five patients were treated with prostacyclin in the period before the decision on transplanting strategy (table 2). Four proceeded to orthotopic transplantation and one was listed for heart-lung transplantation. Mean duration of treatment was 58 (18) days (range 36-78 days). Mean TPG at baseline before the start of prostacyclin was $27(9.8) \mathrm{mm} \mathrm{Hg}$ and after treatment was $17(6.7) \mathrm{mm} \mathrm{Hg}(\mathrm{p}<0.05)$. After administration of vasodilators at cardiac catheterisation, TPG fell from a mean of $19(6.8)$ at the first study to $11(7.5) \mathrm{mm} \mathrm{Hg}$ after treatment $(p<0.05)$. Similarly the mean change in PVRI before and after treatment at baseline was $4.4 \mathrm{WU} \cdot \mathrm{m}^{2}$; this result was not significant, as not all had PVRI measured due to the lack of availability of the mass spectrometer (fig l). Mean pulmonary artery pressure was significantly reduced after prostacyclin administration (51 (12.7) $v 4$ ll (9.0) $\mathrm{mm} \mathrm{Hg}$ ) without a significant rise in left atrial pressure.

\section{Post-transplantation complications}

Twelve orthotopic and two heterotopic transplantations were performed. Two patients were listed for heart-lung transplant.

\section{Orthotopic}

Of the patients receiving an orthotopic transplant, five had complications in the postoperative period. Acute right heart failure occurred in two patients requiring urgent repeat transplantation. Both were bridged to repeat transplantation with ECMO. Three recipients were assisted with mechanical support early after transplantation. One patient made a good recovery with RVAD support. Another required ECMO support despite favourable haemodynamic function preoperatively. The third patient, having required ECMO support preoperatively, received further ECMO after transplantation. He died at 49 days during the post-transplantation intensive care admission of right ventricular failure. The group with these complications and the remaining eight did not differ significantly with respect to time from diagnosis to pretransplantation, TPG, or pulmonary vascular resistance.

\section{Heterotopic}

Two transplants were heterotopic because of pulmonary hypertension. One patient died in the first 30 days. This patient had a difficult postoperative course with poor right ventricular function, presumably related to the pulmonary hypertension, and she eventually developed sepsis while being treated with ECMO. The other patient, who developed accelerated coronary vasculopathy of the heterotopic graft, possibly related to undiagnosed recurrent rejection, died within a year.

\section{DISCUSSION}

The management of paediatric RCM presents a difficult clinical problem. The nature of the disease leads to a rise in pulmonary vascular resistance. This is known to increase the risk of death from right heart failure after orthotopic cardiac transplantation, with a PVRI greater than 5 or $6 \mathrm{WU} \cdot \mathrm{m}^{2}$ increasing the relative risk of death by threefold to fivefold. ${ }^{11-}$ ${ }^{13}$ The rate at which pulmonary vascular disease develops in paediatric RCM is faster than in dilated cardiomyopathy, requiring transplantation at a younger age. ${ }^{14}$ It may be argued that to reduce the risk transplantation should be performed early in the disease, but referring a relatively well child for transplantation needs careful consideration. The decision is complicated by the individual variation in the natural history of the condition. Patients may continue for years with minimal symptoms and others will develop pulmonary vascular disease in a short time. ${ }^{6-9}$ We report on children who have deteriorated quickly and others that have remained asymptomatic for longer periods. Pulmonary vascular resistance may be too high for orthotopic transplantation even before the onset of symptoms. It can also be complicated by associated lesions-the TPG fell dramatically in one of our patients who had an ASD closed between measurements. If pulmonary hypertension persists, a small ASD may be of benefit to decompress the right heart. In one case in our series, an intraoperative ASD was created that may have facilitated event-free recovery.

The timing of transplantation must be guided by the natural history of this disease. Although some children will remain well for many years, a previous report on children with RCM showed progression to a low cardiac output state with left ventricular failure leading to death or transplantation within $2.0(2.3)$ years. ${ }^{15}$ This is similar to our findings 


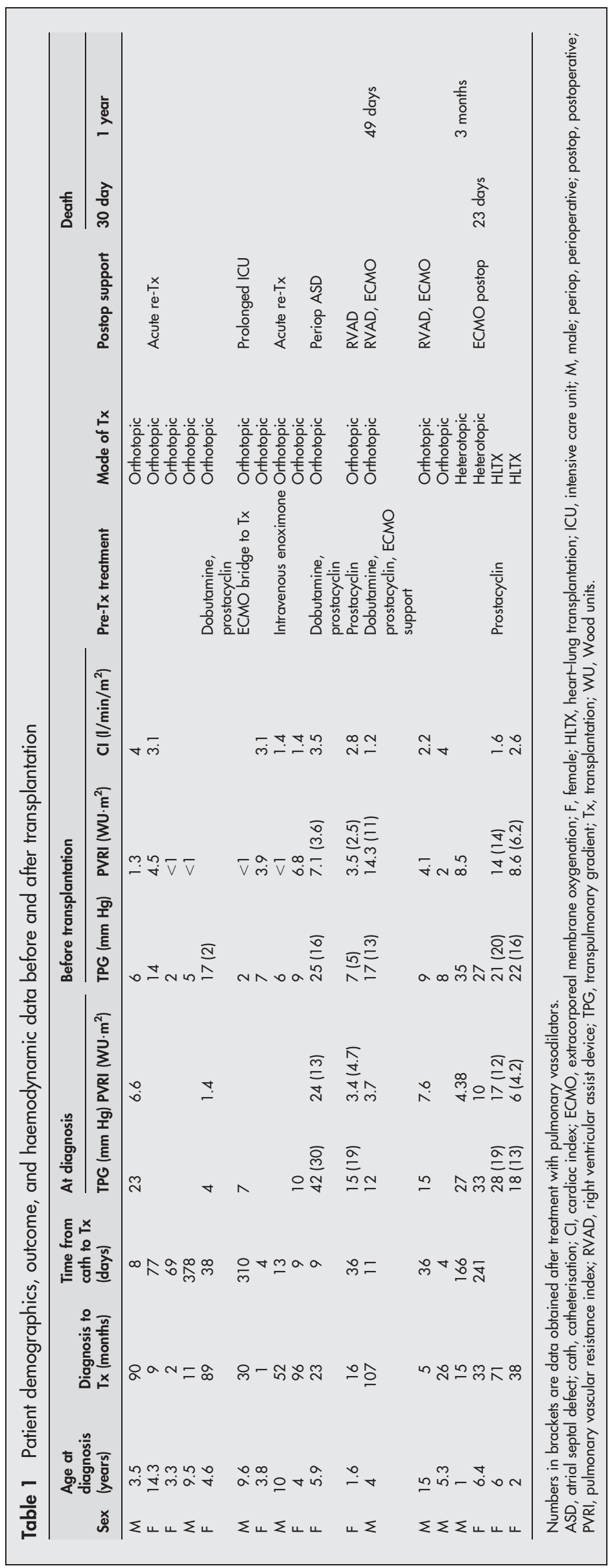

with a mean period of 3.4 (3.2) years between diagnosis and transplantation. It seems unwise to wait until haemodynamic compromise considering the difficult postoperative course for our three patients with cardiovascular collapse. In dilated cardiomyopathy, pulmonary hypertension, when combined with haemodynamic decompensation, raises the relative risk of death to $4.08 .^{11}$

The dilemma with respect to pre-transplantation management is posed by the well child with moderately raised PVRI $\left(4-5 \mathrm{WU} \cdot \mathrm{m}^{2}\right)$. While the patient remains asymptomatic it is difficult to list him or her for transplantation, but as the pulmonary haemodynamic function approaches a level leading to increased risk, we would lean towards offering early orthotopic transplantation in this scenario. For a child with low PVRI we would advocate a strategy of close follow up with regular assessment of pulmonary haemodynamic function, offering transplantation with rising PVRI or worsening symptoms.

Within our series of 14 orthotopic transplantations, two patients had acute graft failure and one required ECMO support for acute right heart failure in the immediate postoperative period. These complications occurred despite favourable pre-transplantation haemodynamic function. A similar sized series of orthotopic transplantations for dilated cardiomyopathy would be unlikely to include two early graft failures. Although this may not be statistically validated it seems reasonable to infer that patients with RCM are a higher risk group for orthotopic transplantation. Conversely, it may be that our haemodynamic measurements before transplantation do not accurately reflect the increased risk of this group of patients.

Only two patients had a severe rise in PVRI/TPG during follow up. This may reflect the use of pretransplantation prostacyclin. There is a paucity of data on the use of pre-transplantation prostacyclin to lower pulmonary vascular resistance before transplantation and we are not aware of any paediatric series reported. In our patients after treatment such therapy was associated with a significant reduction in TPG both with and without acute vasodilator testing in the catheterisation laboratory. The observed change in TPG was associated with a significant reduction in mean pulmonary artery pressure without an important rise in left atrial pressure contributing to the reduction. However, one patient did have a significant rise in left atrial pressure on vasodilator testing during the prostacyclin treatment. Assessment of these patients without considering the haemodynamic variables contributing to the TPG may be misleading. This approach is particularly important in RCM, where the left ventricle may fail to accommodate the increase in blood flow caused by pulmonary vasodilators, leading to high left sided pressures. Our data are encouraging when the fall in mean pulmonary artery pressure is considered in the majority of patients.

Prostacyclin did not always reduce the TPG to less than $14 \mathrm{~mm} \mathrm{Hg}$ and in one patient it was unsuccessful at reversing pulmonary hypertension; we then adopted a heart-lung transplantation strategy. The treatment clinically improved this patient, who is still taking it for symptomatic relief before heart-lung transplantation. In 
Table 2 Outcome of TPG before and after treatment with prostacyclin in five patients

\begin{tabular}{|c|c|c|c|c|c|c|c|}
\hline & 1 & 2 & 3 & 4 & 5 & Mean (SD) & p Value ${ }^{*}$ \\
\hline Duration of treatment before repeat catheterisation (days) & 51 & 49 & 36 & 78 & 75 & $58(18)$ & \\
\hline \multirow{2}{*}{\multicolumn{8}{|c|}{$\begin{array}{l}\text { Before long term prostacyclin treatment } \\
\text { At catheterisation baseline }\end{array}$}} \\
\hline & & & & & & & \\
\hline Mean PA pressure $(\mathrm{mm} \mathrm{Hg})$ & 61 & 66 & 36 & 41 & 50 & $51(12.7)$ & \\
\hline Mean LA pressure $(\mathrm{mm} \mathrm{Hg})$ & 33 & 24 & 21 & 18 & 22 & $24(5.7)$ & \\
\hline TPG $(\mathrm{mm} \mathrm{Hg})$ & 28 & 42 & 15 & 23 & 28 & $27(9.8)$ & \\
\hline $\mathrm{Cl}\left(1 / \mathrm{min} / \mathrm{m}^{2}\right)$ & & 1.7 & 5.5 & 1.9 & 1.6 & & \\
\hline \multicolumn{8}{|l|}{ With acute vasodilator testing } \\
\hline Mean PA pressure $(\mathrm{mm} \mathrm{Hg})$ & 43 & 58 & 31 & 34 & 42 & $42(10.5)$ & \\
\hline Mean LA pressure $(\mathrm{mm} \mathrm{Hg})$ & 33 & 28 & 15 & 16 & 23 & $23(7.7)$ & \\
\hline TPG $(\mathrm{mm} \mathrm{Hg})$ & 11 & 30 & 16 & 18 & 19 & $19(6.8)$ & \\
\hline $\mathrm{Cl}\left(1 / \mathrm{min} / \mathrm{m}^{2}\right)$ & & 2.3 & $5.5 \dagger$ & 1.9 & 1.6 & & \\
\hline \multicolumn{8}{|l|}{ During long term prostacyclin treatment } \\
\hline \multicolumn{8}{|l|}{ At catheterisation baseline } \\
\hline Mean PA pressure (mm Hg) & 48 & 50 & 29 & 34 & 42 & $41(9.0)$ & $<0.01$ \\
\hline Mean LA pressure $(\mathrm{mm} \mathrm{Hg})$ & 31 & 25 & 20 & 17 & 20 & $23(5.5)$ & 0.14 \\
\hline TPG $(\mathrm{mm} \mathrm{Hg})$ & 17 & 25 & 9 & 17 & 22 & $17(6.7)$ & 0.01 \\
\hline $\mathrm{Cl}\left(1 / \mathrm{min} / \mathrm{m}^{2}\right)$ & & 3.5 & 2.6 & 1.2 & 1.6 & & \\
\hline \multicolumn{8}{|l|}{ With acute vasodilator testing } \\
\hline Mean PA pressure $(\mathrm{mm} \mathrm{Hg})$ & 44 & 38 & 26 & 33 & 38 & $35.8(6.7)$ & 0.19 \\
\hline Mean LA pressure $(\mathrm{mm} \mathrm{Hg})$ & 43 & 22 & 19 & 20 & 17 & $24.2(10.7)$ & 0.722 \\
\hline TPG $(\mathrm{mm} \mathrm{Hg})$ & 2 & 16 & 7 & 13 & 21 & $11(7.5)$ & $<0.05$ \\
\hline $\mathrm{Cl}\left(1 / \mathrm{min} / \mathrm{m}^{2}\right)$ & & 4.7 & 2.8 & 1.2 & 1.5 & & \\
\hline
\end{tabular}

another patient PVRI did not fall below $10 \mathrm{WU} \cdot \mathrm{m}^{2}$ despite prostacyclin treatment at home. Although further treatment was planned, the child had a haemodynamic collapse, requiring ECMO bridge to transplantation. A successful outcome was achieved for a patient with a TPG of $25 \mathrm{~mm} \mathrm{Hg}$ (reversible to $16 \mathrm{~mm} \mathrm{Hg}$ ), but RVAD support was required for a TPG that reversed from 15 to $7 \mathrm{~mm} \mathrm{Hg}$ after treatment. Despite the encouraging reduction, it appears that high resistances still preclude safe orthotopic transplantation.

Heterotopic transplantation has been successfully reported for children but was disappointing in our hands. ${ }^{16}$ We have found as in a previous report that right heart failure from high PVRI can still occur with this operation, as it offers left ventricular unloading rather than right ventricular assist. ${ }^{17}$ The shortage of infant donors rather precludes the use of this operation, as small hearts are required for heterotopic donation because of the limited space in the right chest. The increasing pool of small children awaiting a suitable donor in an orthotopic position makes the use of an organ in a heterotopic position unlikely. This is due to both the higher risk associated with a patient requiring a heterotopic

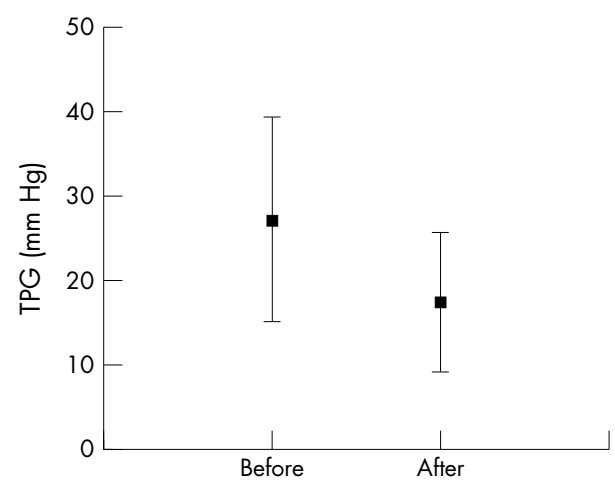

Figure 1 Change in transpulmonary gradient (TPG) after intravenous prostacyclin administration. transplantation and lack of familiarity with both operative techniques and postoperative management.

Improved expertise in paediatric intensive care, particularly with respect to mechanical support devices (ECMO and RVAD), has encouraged transplant physicians to consider the greater potential for long term survival by offering cardiac rather than heart-lung transplantation, as the latter has significantly reduced longer term survival. ${ }^{18}$ It is important, however, to balance the increased short term risk with equivalent survival to cardiac transplantations for other diagnoses in the medium to long term. For this reason combining current pre-transplantation treatment with improvements in intensive care management and postoperative mechanical support may, despite significant perioperative morbidity and mortality, provide a longer overall survival than alternative strategies.

\section{Conclusion}

This, the largest report of paediatric cardiac transplantations for idiopathic RCM, illustrates that it remains a difficult clinical problem. While it is true that some patients remain well for many years, the mean time to transplantation for our patients was less than 3.5 years and for those who were left until haemodynamic compromise occurred, morbidity and mortality increased. This implies that an early transplantation strategy seems prudent. Increase of pulmonary pressure is of concern in this disease, yet we have shown that it may be modulated with prolonged intravenous prostacyclin use. When this is considered, with the advances in mechanical support and paediatric intensive care, orthotopic transplantation may offer a better alternative to heart-lung transplantation for some recipients with moderate pulmonary hypertension.

\section{Authors' affiliations}

M J Fenton, H Chubb, A M McMahon, P Rees, M J Elliott, M Burch, Cardiothoracic Transplant, Great Ormond Street Hospital for Children, London, UK

Competing interest statement: No competing interests are declared from the authors of this study. 
Ethical approval: Ethical approval for this study has been approved from the Institute of Child Health and Great Ormond Street Hospital Ethics Committee, London.

\section{REFERENCES}

1 Lipshultz SE, Sleeper LA, Towbin JA, et al. The incidence of pediatric cardiomyopathy in two regions of the United States. N Engl J Med 2003;348:1647-55

2 Nugent AW, Daubeney PE, Chondros P, et al. The epidemiology of childhood cardiomyopathy in Australia. N Engl J Med 2003;348: 1639-46.

3 Rapezzi C, Ortolani P, Binetti G, et al. Idiopathic restrictive cardiomyopathy in the young: report of two cases. Int J Cardiol 1990;29:121-6.

4 Richardson P, McKenna W, Bristow M. Report of the 1995 World Health Organization/International Society and Federation of Cardiology task force on the definition and classification of cardiomyopathies. Circulation 1996:93:841-2

5 Denfield SW. Sudden death in children with restrictive cardiomyopathy. Card Electrophysiol Rev 2002;6:163-7.

6 Kimberling MT, Balzer DT, Hirsch R, et al. Cardiac transplantation for pediatric restrictive cardiomyopathy: presentation, evaluation, and short-term outcome. J Heart Lung Transplant 2002;21:455-9.

7 Weller RJ, Weintraub R, Addonizio $\amalg$, et al. Outcome of idiopathic restrictive cardiomyopathy in children. Am J Cardiol 2002;90:501-6.

8 Chen SC, Balfour IC, Jureidini S. Clinical spectrum of restrictive cardiomyopathy in children. J Heart Lung Transplant 2001;20:90-2.
9 Cetta F, O'Leary PW, Seward JB, et al. Idiopathic restrictive cardiomyopathy in childhood: diagnostic features and clinical course. Mayo Clin Proc 1995;70:634-40.

10 Kirklin JK, Young JB, McGiffin DC. Recipient evaluation and selection. In: Heart transplantation. Edinburgh: Churchill Livingstone, 2002:210-1.

11 Addonizio L, Hsu DT, Fuzesi L, et al. Optimal timing of pediatric heart transplantation. Circulation 1989;80:11184-9.

12 Carrier M, Rivard M, Latter D, et al. Predictors of early mortality after heart transplantation: the Canadian transplant experience from 1981 to 1992. The CASCADE Investigators. Canadian study of cardiac transplant atherosclerosis determinants. Can J Cardiol 1998;14:703-7.

13 Murali S, Kormos RL, Uretsky BF, et al. Preoperative pulmonary hemodynamics and early mortality after orthotopic cardiac transplantation: the Pittsburgh experience. Am Heart J 1993;126:896-904.

14 Hughes ML, Kleinert S, Keogh A, et al. Pulmonary vascular resistance and reactivity in children with end-stage cardiomyopathy. J Heart Lung Transplant 2000;19:701-4.

15 Kimberling MT, Balzer DT, Hirsch R, et al. Cardiac transplantation for pediatric restrictive cardiomyopathy: presentation, evaluation, and short-term outcome. J Heart Lung Transplant 2002;21:455-9.

16 Khaghani A, Santini F, Dyke CM, et al. Heterotopic cardiac transplantation in infants and children. J Thorac Cardiovasc Surg 1997;113:1042-8.

17 Gamba A, Fiocchi R, Mamprin F, et al. [Heterotropic heart transplantation in young patients with elevated pulmonary vascular resistance]. G Ital Cardiol 1999:29:918-24.

18 Boucek MM, Edwards LB, Keck BM, et al. The Registry of the International Society for Heart and Lung Transplantation: sixth official pediatric report2003. J Heart Lung Transplant 2003;22:636-52.

\section{IMAGES IN CARDIOLOGY}

\section{Right coronary artery embolism from a thrombus of the ascending aorta}

A 52 year old man was admitted for acute inferior myocardial infarction evolving for two hours. He was a mild active smoker, and had no history of heart disease. Coronary angiography revealed occlusions of both the posterior interventricular and retroventricular branches of the right coronary artery (RCA) (panel A). Because of the angiographic appearance, coronary embolism was suspected and a transoesophageal echocardiographic (TOE) examination was performed in search of a cardiac source of the embolism. TOE revealed the presence of an $18 \times 7 \mathrm{~mm}$ pedunculated floating mass in the right coronary sinus of Valsalva. The mass was implanted on a small, minimally calcified atheromatous plaque, and had a highly mobile extremity prolapsing into the RCA ostium during diastole (panel B). These features were suggestive of an ascending aortic thrombus complicated with right coronary embolisation. After five days of medical treatment, including unfractionated heparin, aspirin (75 mg/day), clopidogrel (75 mg/day), and high dose atorvastatin $(80 \mathrm{mg} /$ day), a further TOE showed complete disappearance of the thrombus. The patient was discharged from hospital on $\beta$ blocker, antiplatelet agents, and statin treatment. He is being followed up on an outpatient basis.

\footnotetext{
N Amabile

J Quilici

J-L Bonnet

G Habib

gilbert.habib@free.fr
}
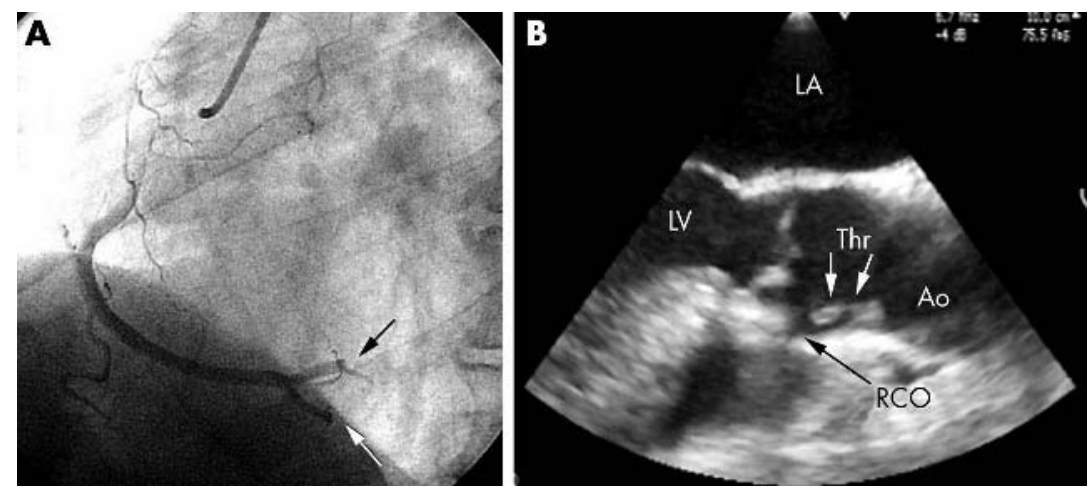

(A) Angiographic data. Right coronary artery selective angiogram (left anterior view) showing TIMI grade 2 flow caused by occlusion of the retroventricular and interventricular branches (arrows). (B) Transoesophageal echocardiographic study. Long axis view showing a mobile pedunculated mass (white arrows) of the ascending aorta. The highly mobile free floating extremity of the thrombus prolapses into the right coronary ostium (RCO, black arrows) during diastole. Ao, aorta; LA, left atrium; LV, left ventricle; RCO, right coronary artery ostium; Thr, thrombus. 